\title{
DIAMETER PROBLEMS FOR UNIVALENT FUNCTIONS WITH QUASICONFORMAL EXTENSION
}

\author{
paul deiermann \\ Department of Mathematics \\ Louisiana State University in Shreveport \\ Shreveport, Louisiana 71115 \\ (Received Apri1 23, 1992 and in revised form September 4, 1992)

\begin{abstract}
This paper utilizes the method of extremal length to study several diameter problems for functions conformal outside of a disc centered at the origin, with a standard normalization, which possess a quasiconformal extension to a ring subdomain of this disc. Known results on the diameter of a complementary component of the image domain of a univalent function are extended. Applications to the transfinite diameters of families of non-overlapping functions and an extension of the Koebe one-quarter theorem are included.
\end{abstract}

KEY WORDS AND PHRASES. Extremal problems, univalent functions, quasiconformal extensions, diameter theorems.

1992 AMS SUBJECT CLASSIFICATION CODES. 30C75, 30C50.

\section{INTRODUCTION}

In this paper we utilize the method of extremal length to study several diameter problems for functions conformal outside of a disc centered at the origin, with a standard normalization, which possess a quasiconformal extension to a ring subdomain of this disc. Similar functions have been studied by many authors, for example, Kühnau [4], Schober [7], and McLeavey [6]. Grötzsch [1] studied diameter problems for normalized quasiconformal functions of subdomains of the unit disc by an unrefined version of extremal length (for easy reference, see Künzi [5]).

The purpose of this paper is to extend known results on the diameter of a complementary component of the image domain of a univalent function. Applications include transfinite diameters of families of non-overlapping functions and an extension of the Koebe one-quarter theorem.

2. NOTATION.

We shall employ the following labels: $z=x+i y, w=u+i v, \zeta=\xi+i \eta, F=U+i V$. Let $\mathbf{D}$ represent the unit disc, $\overline{\mathbf{C}}$ the extended complex plane, $\mathrm{Cl}$ closure and qc. quasiconformal. The Jacobian of a function $F$ is denoted by $J[F]$, and $\Re$ and $\Im$ denote real and imaginary part, respectively. For a simple closed Jordan curve $\gamma$, let $\operatorname{Int}(\gamma)$ and $\operatorname{Ext}(\gamma)$ denote the bounded and unbounded complementary components of $\overline{\mathbf{C}}-\{\gamma\}$, respectively. Multiplication and translation functions are represented by $m_{a}(w)=a w, T_{b}(w)=w-b$, respectively. The following definition of a qc. mapping is used in this study. 
DEFINITION. Let $f$ be an orientation-preserving homeomorphism of a plane domain $G$ which has continuous first partials. Let $D_{f}\left(z_{0}\right)=\left(\left|f_{z}\left(z_{0}\right)\right|+\left|f_{\bar{z}}\left(z_{0}\right)\right|\right) /\left(\left|f_{z}\left(z_{0}\right)\right|-\left|f_{\bar{z}}\left(z_{0}\right)\right|\right)$. If $D_{f}\left(z_{0}\right) \leq K<\infty$ for all $z_{0} \in G$, for some $K \geq 1$, then $f$ is $K$-qc. in $G$. If $D_{f}(z)$ is bounded above by a function $K(|z|) \leq K<\infty$, then $f$ is said to be $K(|z|)$-qc. in $G$.

The following constants appear frequently:

$$
k=(K-1) /(K+1), \quad a_{ \pm}=1 \pm k, \quad B_{ \pm}(K)=2^{-1}(K \pm 1), \quad C_{ \pm}\left(K^{\prime}\right)=B_{ \pm}\left(K^{-1}\right) .
$$

For an arbitrary domain $\mathcal{D}$ that contains infinity, let $\Sigma(\mathcal{D})$ consist of all functions $f$ such that $f$ is univalent and regular in $\mathcal{D}$ apart from a simple pole at infinity where it has the normalized expansion $f(z)=z+b_{0}+b_{1} z^{-1}+\cdots$. For a simple closed Jordan curve $\gamma$ contained in $\mathcal{D}$, the class $\Sigma_{K, \gamma}^{d}(\mathcal{D})$ consists of all functions $f$ such that $f$ restricted to $\mathcal{D} \cap \operatorname{Ext}(\gamma)$ is in $\Sigma(\mathcal{D} \cap \operatorname{Ext}(\gamma)), f$ restricted to $\mathcal{D} \cap \operatorname{Int}(\gamma)$ is $K$-qc., and $f$ is univalent in $\mathcal{D}-\{\gamma\}$. Note that $f$ need not be continuous (or even defined) on $\gamma$, so one could consider $f$ as a pair of "non-overlapping" functions defined on the pair of domains $\mathcal{D} \cap \operatorname{Ext}(\gamma)$ and $\mathcal{D} \cap \operatorname{Int}(\gamma)$. If the $d$ (for discontinuous) is dropped from the symbol for the above class of functions, then we require continuity on $\gamma$. It is clear that $\Sigma_{K, \gamma}(\mathcal{D}) \subset \Sigma_{K, \gamma}^{d}(\mathcal{D})$. As a special case, if $\gamma$ is the circle $C_{R}=\{|z|=R\}$, then let $\Sigma_{K, R}^{*}$ represent the family $\Sigma_{K, C_{R}}(\overline{\mathbf{C}}-\mathrm{Cl}(\mathbf{D}))$.

Modules of a curve family in a domain $\mathcal{D}$ will be used in this study, and will be denoted by $m(\mathcal{D})$ when the curve family has been specified. For reference to this item, see Jenkins [2]. The following canonical mappings are also used. For a domain $\mathcal{D}$ of finite connectivity which contains the origin, let $\Phi_{1}$ denote the circular slit disc mapping which fixes the origin and with $\Phi_{1}^{\prime}(0)>0$, and for a domain of finite connectivity which has at least two boundary continua, let $\Phi_{2}$ represent "the" circular slit ring mapping, which is unique up to rotations. We also use the radial slit disc mapping which fixes the origin and has positive first derivative at the origin. The existence of these mappings is well-known, and our reference will be Jenkins [2], theorems 5.4, 5.6, and 5.5, respectively, pages 74-75.

3. DIAMETER THEOREMS.

THEOREM 1. Let $\mathcal{D}$ be a domain on the $w$-sphere which contains the point at infinity with boundary components $E_{1}=C l(D), E_{2}, \ldots, E_{n}$, where the $E_{j}$, for $j=2, \ldots, n$, are slits on concentric circles centered at the origin. Let $f$ be univalent in $\mathcal{D}, K$-qc. in $\mathcal{D}_{1}=\mathcal{D} \cap\{1<|w|<\rho\}$, regular in $\mathcal{D}_{0}=\mathcal{D} \cap\{|w|>\rho\}$ apart from a simple pole at infinity where it has the expansion

$$
f(w)=\alpha w+a_{0}+a_{1} w^{-1}+\cdots .
$$

The function $f$ need not be continuous on $\mathcal{C}_{\rho}=\{|w|=\rho\}$, but the complementary component of the image domain of $f$ corresponding to $E_{1}$ must contain $C l(D)$. Then $|\alpha| \geq \rho^{1 / K-1}$, with equality if and only if $f$ is equal to

$$
A_{\psi_{1}, \psi_{2}}(w)= \begin{cases}e^{i \psi_{1}} \rho^{1 / K-1} w & \text { for }|w|>\rho, \\ e^{i \psi_{2}} w^{C_{+}(K)} \bar{w}^{C_{-}(K)} & \text { for } 1<|w|<\rho,\end{cases}
$$

where $\psi_{1}, \psi_{2} \in \mathbf{R}$.

PROOF. First we note that, since our goal is to minimize $|\alpha|$, we may restrict our mappings $f$ to those such that $E_{1}^{\prime}=C l(D)$ and where there are no "gaps" between $\mathcal{D}_{1}^{\prime}$ and $\mathcal{D}_{0}^{\prime}$, where image domains and complementary components are denoted by primes. This statement arises from an application of the Riemann mapping theorem combined with Schwarz's inequality.

Let $\zeta(w)=\log (w)$, where the branch chosen is that which takes imaginary values betwen 0 and $2 \pi$. First we cut off a neighborhood of infinity. Let $\mathcal{D}(R)=\mathcal{D} \cap\{|w|<R\}$, where $R$ is chosen large 
enough so that the boundary components of $\mathcal{D}$ are contained in $\mathcal{D}(R)$. Next one transfers to the logarithmic plane via the mapping $\zeta$. Let $\Delta(R)=\zeta(\mathcal{D}(R)), \Delta_{1}=\zeta\left(\mathcal{D}_{1}\right)$, and $\Delta_{0}(R)=\Delta(R)-C l\left(\Delta_{1}\right)$.

The function $f$ induces a mapping $\omega=F(\zeta)$ on the variable $\zeta$ as follows: start with a value of $\zeta \in \Delta(R)$, take the corresponding value of $w$, perform the mapping $f(w)=w^{*}$, then map this point by the same branch of $\zeta$. A calculation reveals that, on the segment $\sigma_{R}=\{(\xi, \eta): 0 \leq \eta<2 \pi, \xi=\log (R)\}$,

$$
F(\zeta)=\zeta+\log (|\alpha|)+O\left(e^{-R}\right) .
$$

We estimate the module of $\Delta^{\prime}(R)$ for the class of curves which separate the boundary component $E_{1}^{\prime}$ from the image of $\sigma_{R}$ under $F$.

For a lower bound, we employ a slight extension of Grötzsch's lemma (see Jenkins[2], theorem 2.6, page 22). In our case, the domains $\Delta^{\prime}(R), \Delta_{1}^{\prime}$, and $\Delta_{0}^{\prime}(R)$ are not doubly-connected due to the additional boundary components. To adapt Grötzsch's lemma to the present situation, instead of employing the canonical mapping of a doubly-connected domain onto a circular ring, as in the proof of Grötzsch's lemma, one must employ the circular slit ring mapping $\Phi_{2}$. With this modification, the rest of the proof of Grötzsch's lemma carries through. After this, we apply the quasi-invariance of the module under a $K$-qc. mapping and conformal invariance of the module to show that

$$
\begin{aligned}
m\left(\Delta^{\prime}(R)\right) & \geq m\left(\Delta_{1}^{\prime}\right)+m\left(\Delta_{0}^{\prime}(R)\right) \\
& \geq K^{-1} m\left(\Delta_{1}\right)+m\left(\Delta_{0}(R)\right) \\
& =\left(K^{-1}-1\right)(2 \pi)^{-1} \log (\rho)+(2 \pi)^{-1} \log (R) .
\end{aligned}
$$

To estimate the module from above, the metric $(2 \pi)^{-1}|d \zeta|$ is clearly admissible in the module problem, thus by employing the expansion for $F$ on $\sigma_{R}$ we have

$$
\begin{aligned}
m\left(\Delta^{\prime}(R)\right) & \leq(2 \pi)^{-2} \iint_{\Delta^{\prime}(R)} d A \\
& \leq(2 \pi)^{-1}\left[\log (|\alpha|)+\log (R)+O\left(e^{-R}\right)\right] .
\end{aligned}
$$

Combining the upper and lower estimates and letting $R$ tend to infinity yields the inequality of the theorem.

The equality statement is obtained by first assuming equality holds throughout the proof and then employing the equality statement in Grötzsch's (extended) lemma. For $w \in \mathcal{D}_{0}$, the uniqueness of the circular slit mapping for domains of finite connectivity (theorem 5.4, page 74, Jenkins [2]) is used, and for $w \in \mathcal{D}_{1}$, one proceeds as in Künzi [5], page 100, Satz 3a.

We remark that the proof of the inequality in theorem 1 only depends upon the modules of $\Delta_{1}$ and $\Delta_{0}(R)$. Thus the inequality remains valid for domains of infinite connectivity, provided the set of boundary slits do not affect the modules of $\Delta_{1}$ and $\Delta_{0}(R)$, i.e., the set of boundary slits form a minimal slit set (for reference, see Jenkins [2], page 81). The price one pays for this extension is a sacrifice of uniqueness of the extremal mapping, since the circular slit mapping need not be unique for domains of infinite connectivity.

We mention another extension of theorem 1. Its proof is an obvious modification of the proof of theorem 1. For theorem 2, $q$ will always represent any integer between 1 and $N$, inclusive, for $N$ any fixed integer. Let $1=R_{0}<R_{1}<\cdots<R_{N}$, denote $C_{R_{q}}=\left\{|w|=R_{q}\right\}$, and let $\mathcal{D}_{q}=\left\{R_{q-1}<|w|<R_{q}\right\} \cap \mathcal{D}$, where $\mathcal{D}$ is as in theorem 1 .

THEOREM 2. Let $\mathcal{D}$ be a domain conditioned as in theorem 1. Let $f$ be as in theorem 1 , but now assume $f$ is $K_{q}$-qc. in $\mathcal{D}_{q}, f$ not necessarily continuous on $C_{R_{q}}$. Then

$$
|\alpha| \geq \frac{1}{R_{N}} \prod_{q=1}^{N}\left(R_{q} / R_{q-1}\right)^{1 / K_{q}}
$$


with equality if and only if $f(w)=A_{\psi_{1}, \psi_{N+1}}(w)$, where

$$
A_{\psi_{1}, \quad, \psi_{N+1}}(w)= \begin{cases}\left.e^{i \psi_{q}} R_{q-1}^{-1 / K_{\iota}} \prod_{j=1}^{q-1}\left(R, / R_{-1}\right)^{1 / K_{,}}{ }_{w} C_{+}\left(K_{q}\right) \bar{w}_{-} C_{-} K_{q}\right) & \text { for } w \in \mathcal{D}_{q}, \\ e^{i \psi_{N+1}} R_{N}^{-1} \prod_{j=1}^{N}(R, / R,-1)^{1 / K}, w & \text { else, }\end{cases}
$$

where $\psi_{1}, \ldots, \psi_{N+1} \in \mathbf{R}$.

The next corollary is proven by forming a Riemann sum and applying the above theorem. For an example of this, see McLeavey [6], and for another method of proof, see Kunzi [5], page 27.

COROLLARY. Let $\mathcal{D}$ be a domain conditioned as in theorem 1. Let $f$ be as in theorem 1 , but now assume that $f$ is $K(|w|)$-qc. in $\mathcal{D}_{1}=\{1<|w|<\rho\} \cap \mathcal{D}$, where for simplicity, we assume that $K(|w|)$ is a continuous function. Then

$$
|\alpha| \geq \rho^{-1} \exp \left[\int_{r=1}^{r=\rho} \frac{1}{K(r)} \frac{d r}{r}\right]
$$

with equality if and only if, for $w=r_{0} e^{i \varphi}, \psi_{1}, \psi_{2} \in \mathbf{R}$,

$$
f(w)= \begin{cases}\exp \left[\int_{r=1}^{r=r_{0}} \frac{1}{K(r)} \frac{d r}{r}\right] e^{i \varphi} e^{i \psi_{1}} & \text { for } 1<r_{0}<\rho, \\ w e^{i \psi_{2}} \rho^{-1} \exp \left[\int_{r=1}^{r=\rho} \frac{1}{K(r)} \frac{d r}{r}\right] & \text { for } r_{0}>\rho .\end{cases}
$$

Now we use theorem 1 to extend a classical diameter theorem due to Grötzsch, where our reference will be Jenkins [2], page 90, theorem 6.5. We follow the notation of Jenkins whenever possible. It will be clear that corresponding results could be proven using theorem 2 in place of theorem 1.

THEOREM 3. Let $D$ be a domain in the $z$-sphere which contains the point at infinity with boundary components $C_{1}, \ldots, C_{n}$, with $n \geq 1$. Let $\gamma$ denote a simple closed Jordan curve contained in $D$, with $C_{1}, \ldots, C_{p} \subset \operatorname{Int}(\gamma)$, for $n \geq p \geq 1$. Let $f^{*} \in \Sigma_{K, \gamma}^{d}(D)$, and let a continuum $\Gamma$ correspond to $C_{1}$ under $f^{*}$. Then the diameter of $\Gamma$ attains its maximum uniquely for the functions $\epsilon_{K}(z)+c$ and its rotations, where $c$ is an arbitrary constant, and $\epsilon_{K}(z)$ is constructed below.

PROOF. Let $D^{*}=D \cap \operatorname{Int}(\gamma), D^{* *}=D \cap \operatorname{Ext}(\gamma)$. One first maps $D^{*}$ conformally onto a circular slit ring with $C_{1}$ corresponding to the circle $|w|=1, \gamma$ corresponding to the circle $|w|=P^{-1}$, for $0<P<1$, and the remaining boundary components in $D^{*}$ corresponding to slits on circles centered at the origin. This mapping is $\Phi_{2}^{*}(z)=1 / \Phi_{2}(z)$, and is unique up to a rotation about the origin.

Next one maps $D^{* *}$ conformally onto $|w|>1 / P$ minus slits on circles centered at the origin, where $\gamma$ corresponds to $|w|=1 / P$, and infinity remains fixed. The mapping is $\Phi_{i}^{*}(z)=\left[P \Phi_{1}\left(z^{-1}\right)\right]^{-1}$, where we assume $\Phi_{1}^{\prime}(0)>0$.

Let $A_{0,0}$ be the function of theorem 1, with $\rho$ replaced by $P^{-1}$, and let $g\left(w^{*}\right)=w^{*}+\left(w^{*}\right)^{-1}$. Let $Q=P^{1 / K} \Phi_{1}^{\prime}(0)$ and define

$$
\epsilon_{K}(z)= \begin{cases}m_{Q} \circ g \circ A_{0,0} \circ \Phi_{1}^{*}(z) & \text { for } z \in D^{* *} \\ m_{Q} \circ g \circ A_{0,0} \circ \Phi_{2}^{*}(z) & \text { for } z \in D^{*}\end{cases}
$$

Let $\Upsilon$ denote the horizontal slit on the real axis which corresponds to $C_{1}$ under the mapping $\epsilon_{K}$. Let $n$ be such that $f^{*}=n \circ \epsilon_{K}$ is an arbitrary function in $\Sigma_{K, \gamma}^{d}(D)$. To apply theorem 1, we must restrict our mappings $n$ to those whose image domains are "geometrically nice."

LEMMA 1. Assume the complementary component $\Upsilon^{\prime}$ of the image domain of $n$ which corresponds to $\Upsilon$ is not a horizontal segment. Let $a, b \in \Upsilon^{\prime}$ be such that $|a-b|$ equals the diameter of $\Upsilon^{\prime}$. 
Then there exists a mapping $h \in \Sigma\left(\overline{\mathbf{C}}-\Upsilon^{\prime}\right)$ such that the complementary component of the image domain of $h$ is a horizontal segment of length $4 \alpha$, for some $\alpha>0$, with $|a-b|<4 \alpha$.

The proof of this lemma follows easily from the Riemann mapping theorem and the classical maximum diameter theorem for univalent functions.

Since $h \circ n \circ \epsilon_{K}$ is a competitor in our problem, to maximize diameter it suffices to assume that $\Upsilon^{\prime}$ is a horizontal segment of length $4 \alpha$, which we temporarily assume lies on the real axis and is centered at the origin. The goal now becomes to maximize $\alpha$, which is accomplished by applying theorem 1 to the function $g^{-1} \circ m_{1 / \alpha} \circ n \circ m_{Q} \circ g \circ A_{0,0}(w)$, where the branch of $g^{-1}$ is that which maps onto the exterior of the closed unit disc, to yield $\alpha \leq Q$.

The uniqueness statement is proven by removing our temporary assumption on $\Upsilon^{\prime}$ and allowing rotations in $\Phi_{1}$ and $\Phi_{2}$, and then renormalizing by rotating the final image domain.

W'hen $\Phi_{i}^{*}$ and $\Phi_{2}^{*}$ are known, one can explicitly calculate the maximum diameter and all extremal functions, as in the following corollary.

COROLLARY 1. Let $f^{*} \in \Sigma_{K, R}^{*}$. The diameter $\rho$ of the complementary component of the image domain of $f^{*}$ satisfies $\varrho \leq 4 R^{1-1 / K}$, with equality if and only if $f^{*}$ equals

$$
f^{E}(z)= \begin{cases}z+\left(R^{2}\right)^{1-1 / K} z^{-1} & \text { for }|z| \geq R, \\ R^{1-1 / K}\left\{|z|^{1 / K-1} z+\left(|z|^{1 / K-1} z\right)^{-1}\right\} & \text { for } 1<|z|<R,\end{cases}
$$

and the rotations and translations $e^{-i 0} f^{E}\left(e^{i 0} z\right)+c$, where $c$ is an arbitrary constant.

A standard manipulation of corollary 1 provides an extension of Koebe's one-quarter theorem.

COROLLARY 2. Let $f^{*}$ be univalent in $\mathbf{D}$, regular in $\{|z|<r\}$ with a continuous $K$-qc. extension to the rest of $\mathrm{D}$, with an expansion in a neighborhood of the origin being $f^{*}(z)=z+a_{2} z^{2}+\cdots$. Also assume that $f^{*}$ does not take the value infinity in $\mathbf{D}$. Then the values of $f^{*}$ cover the disc $\left\{|w|<4^{-1} r^{1-1 / K}\right\}$. If an omitted value $w_{0}$ of $f^{*}$ is such that $\left|w_{0}\right|=4^{-1} r^{1-1 / K}$, then $f^{*}$ equals

$$
f^{E}(z)= \begin{cases}z /\left(1+z^{2} e^{-2 i 0} r^{2(1 / K-1)}\right) & \text { for }|z|<r, \\ \left(r^{-1}|z|\right)^{1 / K-1} z /\left(1+e^{-2 i 0}|z|^{2(1 / K-1)} z^{2}\right) & \text { for } r \leq|z|<1 .\end{cases}
$$

Next an application of corollary 1 is proven which extends corollary 7.3, page 124, in Jenkins [2]. It will be clear that one can also extend corollaries 7.1 and 7.4 in Jenkins [2]. First some labels are given. Let $0<r_{1} \leq 1 \leq r_{2}<\infty$, let $K_{1} \geq 1$, for $i=1,2$, let

$$
\mathcal{D}_{1}=\left\{|z|<r_{1}\right\}, \quad \mathcal{R}_{1}=\left\{r_{1} \leq|z|<1\right\}, \quad \mathcal{D}_{2}=\left\{r_{2}<|z|\right\}, \quad \mathcal{R}_{2}=\left\{1<|z| \leq r_{2}\right\} .
$$

Let $S_{1}$ consist of all functions $f_{1}$ such that $f_{1}$ is $K_{1}$-qc. in $\mathcal{R}_{1}$, with a not necessarily continuous conformal extension to $D$, with expansion for $z \in \mathcal{D}_{1}$ being $f_{1}(z)=a z+a_{2} z^{2}+\cdots$, and $f_{1}$ is nonoverlapping, $f_{1}\left(\left\{\mathcal{R}_{1}\right\}\right) \cap f_{1}\left(\left\{\mathcal{D}_{1}\right\}\right)=0$. Let $\Sigma_{2}$ consist of all functions $f_{2}$ such that $f_{2}$ is $K_{2}$-qc. in $\mathcal{R}_{2}$, with a not necessarily continuous conformal extension to $\bar{C}-\mathrm{Cl}(D)$, with expansion for $z \in \mathcal{D}_{2}$ being $f_{2}(z)=b z+b_{0}+b_{1} z^{-1}+\cdots$, and $f_{2}\left(\left\{\mathcal{R}_{2}\right\}\right) \cap f_{2}\left(\left\{\mathcal{D}_{2}\right\}\right)=0$.

COROLLARY 3. Let $f_{1} \in S_{1}, f_{2} \in \Sigma_{2}$, with $f_{1}(\mathbf{D}) \cap f_{2}(\bar{C}-\mathrm{Cl}(\mathbf{D}))=0$. Then

$$
|a / b| \leq r_{1}^{2 C_{-}\left(K_{1}\right)} / r_{2}^{2 C_{-}\left(K_{2}\right)},
$$

with equality occurring only for the functions, $\varphi_{i}, \psi_{i} \in \mathbf{R}$, for $i=1,2, \mu>0$,

$$
f_{i}^{E}(z)= \begin{cases}z^{C_{+}\left(K_{i}\right) \bar{z} C_{-}\left(K_{i}\right) e^{i \psi_{i} \mu}} & \text { for } z \in \mathcal{R}_{i}, \\ z r_{i}^{2 C_{-}\left(K_{i}\right)} e^{i \varphi_{i} \mu} & \text { for } z \in \mathcal{D}_{i} .\end{cases}
$$


PROOF. The proof follows the same scheme as before. One forms the alleged extremal functions, then composes them with functions such that the compositions are competitors in the extremal problem. The univalent case (Jenkins [2], corollary 7.3) combined with the Riemann mapping theorem allows one to restrict competition to only those functions whose image domains are geometrically nice. An application of corollary 1 then proves the theorem.

We conclude this paper with corresponding results for the minimum diameter theorem. For this we first achieve a result analogous to theorem 1.

THEOREM 4. Let $\mathcal{D}$ be a domain on the $w$-sphere which contains infinity, with boundary components $E_{1}=C l(\mathrm{D}), E_{2}, \ldots, E_{n}$, where the $E_{j}$, for $j=2, \ldots, n$, consist of slits on rays emanating from the origin, and which are contained in $\{1<|w|<\rho\}$. Let $f$ be continuous and univalent in $\mathcal{D}$ with continuous first partials in $\mathcal{D}_{1}=\mathcal{D} \cap\{1<|w|<\rho\}$, regular in $\mathcal{D}_{0}=\mathcal{D} \cap\{|w|>\rho\}$ apart from a simple pole at infinity where it has the expansion (3.1). Assume the complementary component of the image domain of $f$ which corresponds to $E_{1}$ is the closed unit disc. Let $\zeta(w)=\log (w)$ be as in theorem 1 , let $\Delta_{1}=\zeta\left(\mathcal{D}_{1}\right)$, and let $\omega=F(\zeta)$ be the mapping induced by $f$ as in theorem 1. Finally, for $\zeta \in \Delta_{1}$, assume the induced map $F$ satisfies

$$
J[F] \geq 1 /\left(2\left(1+k^{2}\right)\right)\left\{\left(a_{+}\right)^{2}\left|\frac{\partial F}{\partial \xi}\right|^{2}+\left(a_{-}\right)^{2}\left|\frac{\partial F}{\partial \eta}\right|^{2}\right\} .
$$

Then $|\alpha| \leq 1$, with equality if and only if $f$ is a rotation of $\mathcal{D}$.

PROOF. As in theorem 1 we map to the logarithmic plane. We will need uniform convergence of the expansion of $F$, so let $\epsilon>0$, let $\Delta_{\epsilon}$ be the image of $\left\{1<|w|<\rho e^{\epsilon}\right\} \cap \mathcal{D}$ under $\zeta$, and let $\Delta_{\epsilon}^{\prime}$ be the image of $\Delta_{\epsilon}$ under the induced mapping $F$. For $\zeta \in \Delta_{0}=\zeta\left(\mathcal{D}_{0}\right)$, the following expansion is valid for $F$ :

$$
F(\zeta)=\zeta+\log (\alpha)+\text { powers of } e^{-\zeta}
$$

Using this expansion, an upper bound for the area of $\Delta_{\epsilon}^{\prime}$ is given by

$$
\begin{aligned}
A_{\Delta_{\varepsilon}^{\prime}} & \leq \int_{\eta=0}^{\eta=2 \pi} \Re[F(\log (\rho)+\epsilon+i \eta)] d \eta \\
& =2 \pi \log (|\alpha|)+A_{\Delta_{e}},
\end{aligned}
$$

where $A_{S}$ denotes the area of a set $S$.

For a lower bound, first we must restrict our functions to those whose image domains are geometrically nice.

LEMMA 2. Assume the complementary components of the image domain of $f$ corresponding to $E_{2}, \ldots, E_{n}$ do not all consist of slits on rays emanating from the origin. Then there exists a function $h$, regular and univalent in $f(\mathcal{D})$ apart from a simple pole at infinity where $h(W)=\beta W+b_{0}+b_{1} W^{-1}+\cdots$, such that the complementary component of the image domain of $h$ corresponding to the closed unit disc is the closed unit disc, the other complementary components consist of slits on rays emanating from the origin, and furthermore, $|\beta|>1$.

The proof of this lemma is identical to the proof of lemma 1 except now the existence of $h$ is a well-known example of a canonical conformal mapping (see, for example, theorem 5.5, Jenkins [2], page 74). It is also well-known that $|\beta|>1$, strict inequality due to the assumption on the image domain of $f$ (see theorem 5.2, Jenkins [2], page 73).

If we let $g^{*}=h \circ f$, it is easy to see that the mapping induced by $g^{*}$ satisfies inequality (3.2). Thus $g^{*}$ is a competitor of $f$ with a geometrically nice image domain such that $\left|g^{* \prime}(\infty)\right|>\left|f^{\prime}(\infty)\right|$. Therefore 
it suffices to assume that the image domain of $f$ has complementary components consisting of the closed unit disc along with slits on rays emanating from the origin.

The lower bound for the area of $\Delta_{c}^{\prime}$ is now derived. First integrate both sides of (3.2) over $\Delta_{\epsilon}$. Apply Schwarz's inequality and then Fubini's theorem to each of the integrals on the right side of (3.2). After this, employ an obvious geometric inequality, which is where the lemma on the boundary components of the image domain of $f$ comes into play. The details are as follows.

Let $\tau_{\eta_{0}}=\left\{(\xi, \eta): 0<\xi \leq \log (\rho)+\epsilon, \eta=\eta_{0}\right\}$, then

$$
\begin{aligned}
\iint_{\Delta_{\epsilon}}\left|\frac{\partial F}{\partial \xi}\right|^{2} d A & \geq\left(A_{\Delta_{\epsilon}}\right)^{-1}\left\{\int_{\eta=0}^{\eta=2 \pi} \int\left|\frac{\partial F}{\partial \xi}\right| d \xi d \eta\right\}^{2} \\
& \geq\left(A_{\Delta_{\epsilon}}\right)^{-1}\left\{\int_{\eta=0}^{\eta=2 \pi} \Re[F(\log (\rho)+\epsilon+i \eta)-0] d \eta\right\}^{2} \\
& \geq A_{\Delta_{\epsilon}}+(2) 2 \pi \log (|\alpha|),
\end{aligned}
$$

where we only integrate over those $\eta$ 's which avoid the horizontal boundary slits.

Similarly, using vertical segments instead of horizontal segments, we get

$$
\iint_{\Delta_{\epsilon}}\left|\frac{\partial F}{\partial \eta}\right|^{2} d A \geq A_{\Delta_{\epsilon}}
$$

Inserting (3.3) and (3.4) into the integrated inequality obtained from (3.2) yields

$$
\iint_{\Delta_{\epsilon}} J[F] d A \geq A_{\Delta_{\epsilon}}+\left(1+2 k /\left(1+k^{2}\right)\right) 2 \pi \log (|\alpha|) .
$$

Combining the upper and lower estimates yields the inequality of the theorem.

The proof of the equality statement is straightforward.

COROLLARY. Let $f^{*} \in \Sigma_{K, R}^{*}$. The diameter $\varrho$ of the complementary component of $f^{*}$ satisfies $\varrho \geq 2 R^{1-K}$, with equality if and only if $f^{*}$ equals

$$
f^{E}(z)= \begin{cases}z & \text { for }|z| \geq R, \\ R^{1-K_{z^{B}} B^{(K)} \bar{z}^{B_{-}(K)}} & \text { for } 1<|z|<R,\end{cases}
$$

and the translations $f^{E}(z)+c$, where $c$ is an arbitrary constant.

PROOF. Let $f$ be an arbitrary function such that $f^{*}=f \circ m_{R^{K-1}} \circ f^{E} \in \Sigma_{K, R}^{*}$, with an expansion in $\left\{|w|>R^{K}\right\}$ being $f(w)=R^{1-K} w+b_{0}+b_{1} w^{-1}+\cdots$. Another analogue of lemma 1 is needed to restrict our functions to those with geometrically nice image domains. The proof is the same as that of lemma 1, except now one combines the Riemann mapping theorem with the classical minimum diameter theorem for univalent functions.

LEMMA 3. Assume the complementary component $\Upsilon^{\prime}$ of the image domain of $f$ which corresponds to $C l(D)$ is not a circle. Then there exists $h \in \Sigma\left(\bar{C}-\Upsilon^{\prime}\right)$ such that the complementary component of the image domain of $h$ is a circle of diameter $\varrho$. Moreover, if $e^{*}$ is the diameter of $\Upsilon^{\prime}$, then $\varrho<e^{*}$.

By lemma 3, since the goal is to minimize diameter, and since $h \circ f^{*}$ is a competitor, it suffices to assume that $f^{*}$ has complementary component a circle centered at a point $c$, of radius $\alpha$. So minimizing $\alpha$ becomes the goal. A calculation verifies that inequality (2) of theorem 4 holds for the induced mapping $F$ of $f$. Thus an application of theorem 4 to the function $m_{1 / \alpha} \circ T_{-c} \circ f$ proves the corollary. 
ACKNOWLEDGEMENT. This work was done under the supervision of Professor James A. Jenkins of Washington University in St. Louis.

\section{REFERENCES}

[1] GRÖTZSCH, H. Über die Verzerrung bei nichtkonformen schlichten Abbildungen mehrfach zusammenhängender schlichter Bereiche, Berichte d. math.-phys. Klasse d. Sächs. Akad. d. Wiss. zu Leipzig 82, 69-80 (1930).

[2] JENKINS, J.A. Univalent functions and conformal mapping, Springer-Verlag, Berlin-GöttingenHeidelberg, 1958.

[3] JENKINS, J.A. A uniqueness result in conformal mapping, Proc. Amer. Math. Soc. 22 (1969) pp.324-325.

[4] KÜHNAU, R. Verzerrungssätze und Koeffizientenbedingungen vom Grunskyschen Typ für quasikonforme Abbildungen, Math. Nachr. 48 (1971) pp. 77-105.

[5] KÜNZI, H.P. Quasikonforme Abbildungen, Ergebn. d. Math. u. ihrer Grenzgebiete; Neue Folge, H. 26. Berlin-Göttingen-Heidelberg: Springer 1960.

[6] McLEAVEY, J.O. Extremal problems in classes of analytic functions with quasiconformal extensions, Trans. Amer. Math. Soc. 195 (1974) pp. 327-343.

[7] SCHOBER, G. Univalent functions-selected topics, Lecture Notes in Mathematics 478, Springer-Verlag, Berlin-New York, 1975. 


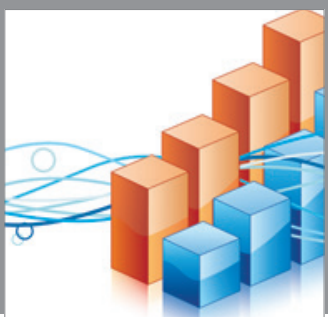

Advances in

Operations Research

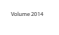

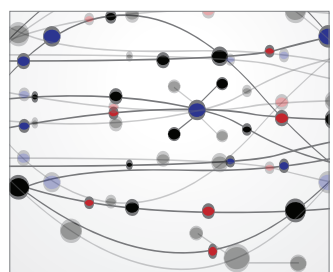

\section{The Scientific} World Journal
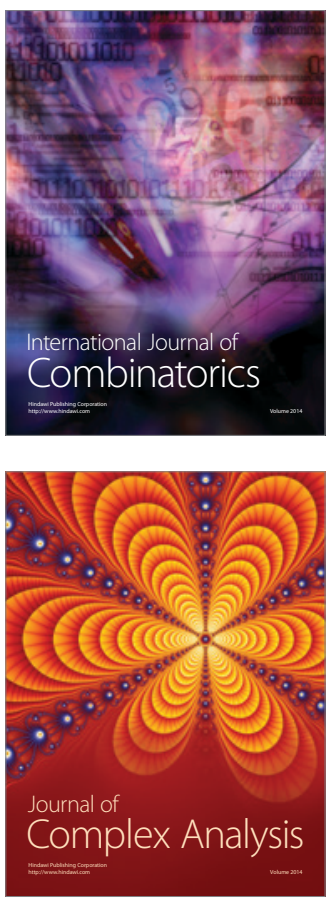

International Journal of

Mathematics and

Mathematical

Sciences
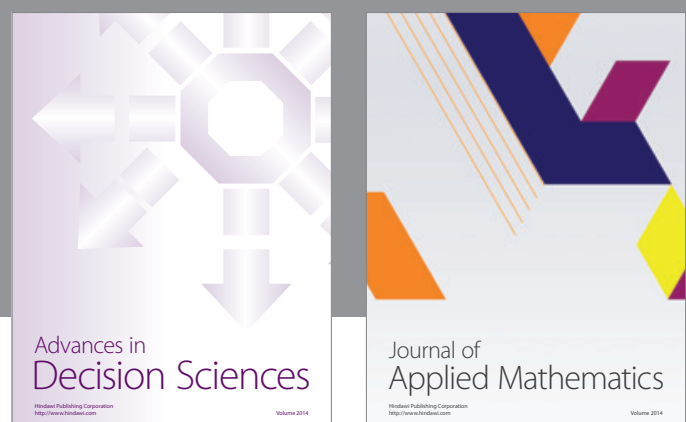

Journal of

Applied Mathematics
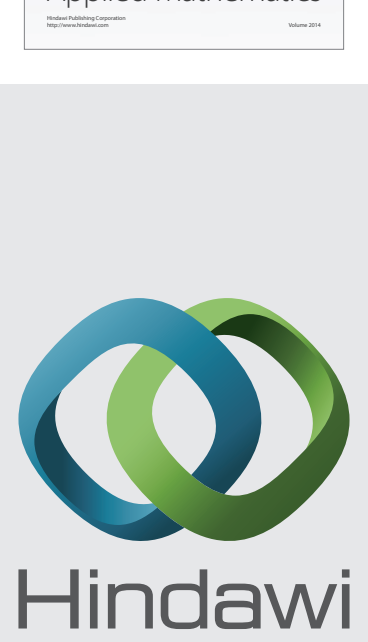

Submit your manuscripts at http://www.hindawi.com
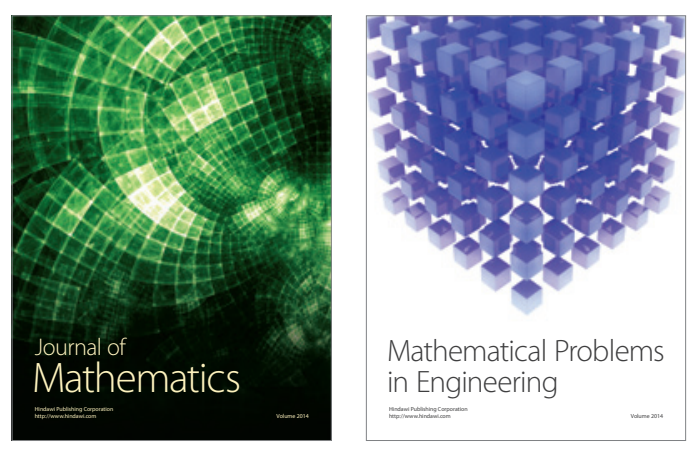

Mathematical Problems in Engineering
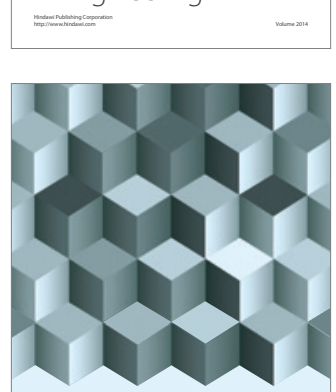

Journal of

Function Spaces
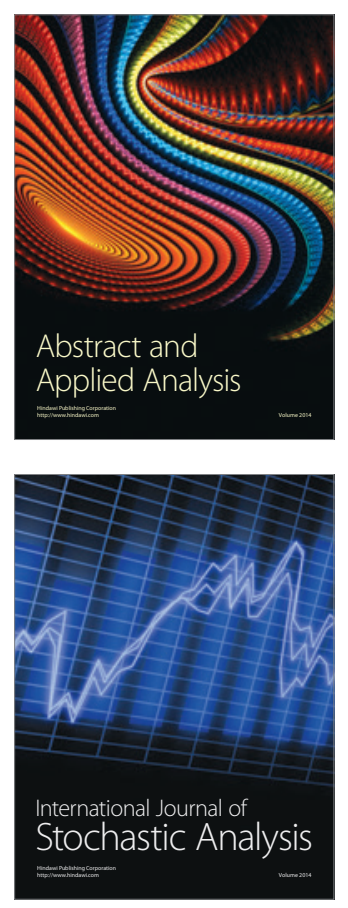

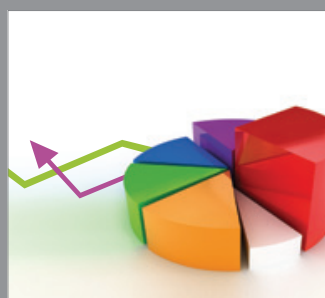

ournal of

Probability and Statistics

Promensencen
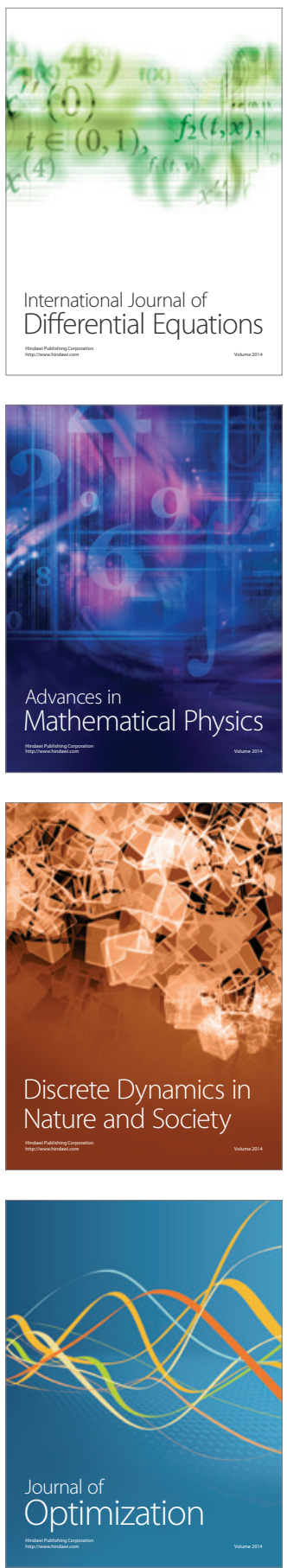